\title{
Common Fixed Point Theorem for Occasionally Converse Commuting Maps in Complex-Valued Metric Space
}

\author{
Rinku Sharma ${ }^{1}$, Dinesh $^{2}$ \\ ${ }^{1}$ Department of Mathematics, University of Delhi, Delhi 110007, India \\ ${ }^{2}$ Department of Mathematics, D.N. College, Hisar 125001, Haryana, India
}

Abstract: In this paper, we proved a common fixed point theorem for occasionally converse commuting (OCC) self-maps without continuity of maps for non-complete complex-valued metric space.

Keywords: Converse commuting maps, occasionally converse commuting maps, fixed point and complexvalued metric space

\section{Introduction}

Lii [2] introduced the concept of converse commuting maps and proved some common fixed point results. Liu and $\mathrm{Hu}$ [3] used this concept for multi-valued mappings. Later on H.K. Pathak and R.K. Verma [6] introduced occasionally converse commuting maps and proved common fixed point theorems for occasionally converse commuting maps in symmetric spaces.

A. Azam, B. Fisher and M. Khan [1] introduced the concept of complex-valued metric space and obtain a common fixed point theorem for a pair of mappings satisfying contractive type condition.

\section{Definitions And Preliminaries}

Let $\square$ be the set of complex numbers and $z_{1}, z_{2} \in \square$. Define a partial order $\llbracket$ on $\square$ as follows:

$z_{1} \rrbracket z_{2}$ if and only if $\operatorname{Re}\left(z_{1}\right) \leq \operatorname{Re}\left(z_{2}\right), \operatorname{Im}\left(z_{1}\right) \leq \operatorname{Im}\left(z_{2}\right)$.

Note that

$$
\begin{aligned}
& 0 \rrbracket z_{1} Æ z_{2} \Rightarrow\left|z_{1}\right|<\left|z_{2}\right| \\
& z_{1} \text { 『 } z_{2}, z_{2} \text { 『 } z_{3} \Rightarrow z_{1} \rrbracket z_{3} .
\end{aligned}
$$

Definition 2.1 ([1]). Let $X$ be a nonempty set. Suppose that the mapping $d: X \times X \rightarrow \square$, satisfies:

(i) 0 I $d(x, y)$, for all $x, y \in X$ and $d(x, y)=0$ if and only if $x=y$;

(ii) $d(x, y)=d(y, x)$, for all $x, y \in X$;

(iii) $d(x, y) \llbracket d(x, z)+d(z, y)$, for all $x, y, z \in X$.

Then $d$ is called a complex-valued metric on $X$, and $(X, d)$ is called a complex-valued metric space.

Example 2. Let $X=\square$ be the set of complex numbers. Suppose that the mapping $d: \square \times \square \rightarrow \square$, defined by

$$
\begin{aligned}
d\left(z_{1}, z_{2}\right)=\left|x_{1}-x_{2}\right|+i\left|y_{1}-y_{2}\right|, \text { for all } & z_{1}, z_{2} \in \square \\
& z_{1}=x_{1}+i y_{1} \\
& z_{2}=x_{2}+i y_{2}
\end{aligned}
$$

Then $(\square, d)$ is a complex-valued metric space.

Definition 2.3 ([2]). A point $x \in X$ is called a commuting point of $f, g: X \rightarrow X$ if $f g x=g f x$.

Definition 2.4 ([2]). Maps $f, g: X \rightarrow X$ are said to be converse commuting if $f g x=g f x$ implies $f x=g x$.

Definition 2.5 ([6]). Two self-maps $f, g: X \rightarrow X$ are said to be occasionally converse commuting, if for some $x$ in $X \quad f g x=g f x$ implies $f x=g x$.

Following example shows that, every conversely commuting mapping is (OCC) but the converse need not be true. 
Example 2.6. Let $X=\{z \in \square: \operatorname{Im}(z) \geq 0, \operatorname{Re}(z)=0\}$ and the self-mapping $f$ and $g$ are defined by:

$f(z)=\left\{\begin{array}{cl}\frac{i}{n+1}, & \text { if } z=\frac{i}{n}, n \in \square \\ 0, & \text { otherwise }\end{array}, \quad g(z)=\left\{\begin{array}{cl}\frac{i}{n+2}, & \text { if } z=\frac{i}{n}, n \in \square \\ 0, & \text { otherwise }\end{array}\right.\right.$

$f g\left(\frac{i}{n}\right)=f\left(\frac{i}{n+2}\right)=\frac{i}{n+3}, g f\left(\frac{i}{n}\right)=g\left(\frac{i}{n+1}\right)=\frac{i}{n+3}$.

But $f\left(\frac{i}{n}\right) \neq g\left(\frac{i}{n}\right)$.

\section{Main Result}

Theorem 3.1. Let $(X, d)$ be a complex valued-metric space and let $f, g, h$ and $k$ be four self-maps defined on $X$, such that the pairs $(f, k)$ and $(g, h)$ are occasionally converse commuting maps satisfying:

$$
d(f x, g y) \text { 『 } \lambda \max \{d(k x, h y), d(f x, k x), d(g y, h y), d(g y, k x), d(f x, h y)\}
$$

for all $x, y \in X, \lambda \in(0,1)$, then $f, g, h$ and $k$ have a unique common fixed point in $X$.

Proof. Let OCC $(f, k)$ denote the set of occasionally converse commuting points of $f$ and $k$. Since the pairs $(f, k)$ is occasionally converse commuting, by definition, there exists some $u \in \operatorname{OCC}(f, k)$; such that $f k u=k f u$ implies $f u=k u$. Hence $d(f u, k u)=0$.

It follows that

$f f u=f k u=k f u$.

Similarly, the occasionally converse commuting points for the pair $(g, h)$ implies that there exists $v \in \mathrm{OCC}(g, h)$ such that $g h v=h g v$ implies $g v=h v$. Hence $d(g v, h v)=0$ and so

$g g u=g h v=h g v$.

First, we prove that $f u=g v$. If not, then using (3.1) for $\mathrm{x}=\mathrm{u}, \mathrm{y}=\mathrm{v}$.

$d(f u, g v)$ ๆ $\lambda \max \{d(k u, h v), d(f u, k u), d(g v, h v), d(g v, k u), d(f u, h v)\}$

I $\lambda \max \{d(f u, g v), 0,0, d(g v, f u), d(f u, g v)\}$

I $\lambda \max \{d(f u, g v)\}$

which implies that

$|d(f u, g v)| \leq \lambda|d(f u, g v)|$, a contradiction.

Therefore $f u=g v$.

Now, we claim that $f f u=f u$. If not, then considering (3.1) for $x=g u, y=v$, we have

$d(f f u, g v) \uparrow \lambda \max \{d(k f u, h v), d(f f u, k f u), d(g v, h v), d(g v, k f u), d(f f u, h v)\}$

ๆ $\lambda \max \{d(f f u, g v), d(f f u, k f u), d(g v, h v), d(g v, k f u), d(f f u, h v)\}$

I $\lambda \max \{d(f f u, g v), 0,0, d(f u, f f u), d(f f u, f u)\}$

I $\lambda \max \{d(f f u, f u)\}$

which implies that

$|d(f f u, f u)| \leq \lambda|d(f f u, f u)|$, a contradiction.

Therefore $f f u=f u$. Similarly $g g v=g v$. Since $f u=g v$, we have

$f u=g v=f f u=f k u=k f u=g g v=h g v=g h v$.

Therefore $f u=w$ (say), is a common fixed point of $f, g, h$ and $k$. For uniqueness, let $w^{\prime} \neq w$ be another common fixed point of $f, g, h$ and $k$, then by (3.1), we have

$d\left(f w, g w^{\prime}\right)$ ๆ $\lambda \max \left\{d\left(k w, h w^{\prime}\right), d(f w, k w), d\left(g w^{\prime}, h w^{\prime}\right), d\left(g w^{\prime}, k w\right), d\left(f w, h w^{\prime}\right)\right\}$

I $\lambda \max \left\{d\left(w, w^{\prime}\right), d(w, w), d\left(w^{\prime}, w^{\prime}\right), d\left(w^{\prime}, w\right), d\left(w, w^{\prime}\right)\right\}$

I $\lambda \max \left\{d\left(w, w^{\prime}\right), 0,0, d\left(w, w^{\prime}\right), d\left(w, w^{\prime}\right)\right\}$

which implies that

$\left|d\left(w, w^{\prime}\right)\right| \leq \lambda\left|d\left(w, w^{\prime}\right)\right|$, a contradiction.

Therefore, $w=f u$ is a unique common fixed point of $f, g, h$ and $k$. 
Corollary 3.2. Let $(X, d)$ be a complex-valued metric space and let $f, k$ be self-maps on $X$ such that the pair $(f, k)$ is occasionally converse commuting maps satisfying

$d(f x, f y) \rrbracket \lambda \max \{d(k x, k y), d(f x, k x), d(f y, k y), d(f y, k x), d(f x, k y)\}$

for all $x, y \in X, \lambda \in(0,1)$, then $(f, k)$ have a unique common fixed point in $X$.

Example 3.3. Let $X=\{z \in \square: 0 \leq \operatorname{Im}(z)<1, \operatorname{Re}(z)=0\}$.

Let $d: X \times X \rightarrow \square$ be the metric, defined by

$d\left(z_{1}, z_{2}\right)=\left|x_{1}-x_{2}\right|+i\left|y_{1}-y_{2}\right|$, for all $z_{1}, z_{2} \in \square$

$$
\begin{aligned}
& z_{1}=x_{1}+i y_{1} \\
& z_{2}=x_{2}+i y_{2}
\end{aligned}
$$

Define the maps $f, g, h$ and $k: X \rightarrow X$ as follows:

$$
\begin{array}{ll}
f(z)=\left\{\begin{array}{cl}
\frac{i}{n+4}, & \text { if } z=\frac{i}{n}, n \in \square \\
0, & \text { otherwise }
\end{array}, \quad g(z)=\left\{\begin{array}{cl}
\frac{i}{n+3}, & \text { if } z=\frac{i}{n}, n \in \square \\
0, & \text { otherwise }
\end{array}\right.\right. \\
h(z)=\left\{\begin{array}{cl}
\frac{i}{n+2}, & \text { if } z=\frac{i}{n}, n \in \square \\
0, & \text { otherwise }
\end{array}, \quad k(z)=\left\{\begin{array}{cl}
\frac{i}{n+1}, & \text { if } z=\frac{i}{n}, n \in \square \\
0, & \text { otherwise }
\end{array}\right.\right.
\end{array}
$$

There exists $u \in X-\left\{\frac{i}{n}: n \in \square\right\}$ such that $f k u=k f u$ implies that $f u=k u$. Hence $(f, k)$ is (OCC). Similarly, $(g, h)$ is (OCC). The set of (OCC) of $f$ and $k$, and $g$ and $h$ are given by

$$
\operatorname{OCC}(f, k)=\operatorname{OCC}(g, h)=u \in X-\left\{\frac{i}{n}: n \in \square\right\} \text {. }
$$

All the conditions of Theorem 3.1 are satisfied.

$f u=k u=g v=h v=0$ is the unique common fixed point of $f, g, h$ and $k$.

\section{References}

[1] A. Azam, B. Fisher and M. Khan, Common fixed point theorems in complex-valued metric spaces, Numerical Functional Analysis and Optimization, 32 (3) (2011), 243-253.

[2] Z. Lii, On common fixed point theorem for converse commuting self maps on metric spaces, Acta Anal. Funct. Appl., 4(3) (2002), 226-228.

[3] Q. Liu and $\mathrm{X}$. Hu, some new common fixed point theorems for converse commuting multivalued maps in symmetric spaces with applications, Nonlinear Analysis Forum, 10(1) (2005), 97-107.

[4] R. Chugh, Sumitra and M. Alamgir Khan, Common fixed point theorems for converse commuting maps in fuzzy metric spaces, Int. Math. Forum, 37(6) (2011), 1183-1190.

[5] H.K. Pathak and R.K. Verma, Integral type contractive condition for converse commuting mappings, Int. J. Math. Anal., 3(24) (2009), 1183-1190.

[6] H.K. Pathak and R.K. Verma, Common fixed point theorems for occasionally converse commuting mappings in symmetric spaces, Kathmandu Univ. J. Sci., Eng. and Tech., 3(1) (2011), 56-62.

[7] M. Abbas and B.E. Rhoades, Common fixed point theorems for occasionally weakly compatible mappings satisfying a generalized contractive condition, Mathematical Communications, 13 (2008), 295-301. 